Article

\title{
New Technologies in Orthodontics: A Digital Workflow to Enhance Treatment Plan and Photobiomodulation to Expedite Clinical Outcomes
}

\author{
Vincenzo Quinzi ${ }^{1}$ (), Vincenzo Ronsivalle ${ }^{2, *}$, Vincenzo Campanella ${ }^{3}$, Leonardo Mancini ${ }^{1}{ }^{(}$, \\ Salvatore Torrisi ${ }^{2}$ and Antonino Lo Giudice ${ }^{2}$ (i) \\ 1 Department of Life, Health \& Environmental Sciences Postgraduate School of Orthodontics, University of \\ L'Aquila, 67100 L'Aquila, Italy; vincenzo.quinzi@univaq.it (V.Q.); mancinileonardo94@gmail.com (L.M.) \\ 2 Department of Orthodontics, School of Dentistry, University of Catania, 95123 Catania, Italy; \\ ture_torrisi@hotmail.it (S.T.); nino.logiudice@gmail.com (A.L.G.) \\ 3 Department of Clinical and Translational Medicine, Tor Vergata University of Rome, 00100 Rome, Italy; \\ vincenzo.campanella@uniroma2.it \\ * Correspondence: vincenzo.ronsivalle@hotmail.it; Tel.: +39-347-86-16-273
}

Received: 11 January 2020; Accepted: 14 February 2020; Published: 21 February 2020

\begin{abstract}
Background: The transversal maxillary deficiency represents one of the most frequent skeletal discrepancies of the craniofacial region. The analysis of morphological characteristics of the maxilla can be detrimental for a correct diagnosis and treatment plan. Methods: This paper shows a user-friendly digital workflow involving mirroring, superimposition, and the deviation analysis of 3D models of the maxilla in order to identify the presence of symmetry/asymmetry of the palatal vault. Such information can be helpful to clinicians in order to design an appropriate orthodontic appliance for the treatment of transversal maxillary deficiency. We also describe a case report of a seven-year-old female affected by mild transversal maxillary deficiency associated with anterior openbite. The appliance is designed after a comprehensive evaluation of the morphology of the maxilla performed by using the presented diagnostic digital workflow. Additionally, the orthodontic treatment is assisted by photobiomodulation sessions that expedite the achievement of clinical outcomes.
\end{abstract}

Keywords: digital dentistry; digital orthodontics; photobiomodulation

\section{Introduction}

Maxillary transverse deficiency (MTD) represents one of the most common skeletal deformities of the craniofacial region [1,2]. Skeletal maxillary expansion, in the form of rapid and slow maxillary expansion protocols (SME) [3], is an efficient therapy for the treatment of MTD. Usually, the diagnosis of MTD is based on visual inspection of posterior-crossibite, despite the fact that transverse dento-alveolar compensation may cause underestimation of this condition [4]. In particular, dento-alveolar compensation may mitigate the negative effect of MTD on the occlusion as well as camouflage specific morphological characteristics of the maxilla such as the asymmetry of the palatal vault and/or of the dento-alveolar processes [5]. The analysis of morphological characteristics of the maxilla is fundamental for a correct diagnosis and treatment plan of MTD. In this respect, the awareness of the symmetry/asymmetry of the maxilla will guide the clinicians in choosing the design of the maxillary expander for each patient $[5,6]$.

To date, progress in 3D imaging has opened new scenarios for the evaluation of anatomical characteristics and morphological changes in dentistry [7,8]. In the orthodontic field, for example, digital scans of maxillary and mandibular jaws can be registered (superimposition) and deviation analysis can be used to detect shape and to obtain detailed dimensional information. Moreover, 3D 
models of the maxilla and/or mandible of the same subject can be mirrored and superimposed in order to detect anatomical asymmetry as well as asymmetrical changes between two sides [9-11]. This information can be provided by referring to "surface-to-surface" analysis, and can be visualized in colors in a 3D color-map [9-11].

Once a correct diagnosis and treatment plan are established, clinicians are concerned about the total treatment time required for reaching clinical outcomes. This is particularly relevant in young patients, when orthodontic therapies should be as efficient as possible in order to maintain patients' compliance. In this respect, photobiomodulation (PBM) represents the new frontier for expediting orthodontic movement, as confirmed by previous clinical studies [12-15]. In particular, photobiostimulation is a noninvasive irradiation procedure that uses a laser light within the red to near-infrared range (wavelengths from 632 to $1064 \mathrm{~nm}$ ) to provoke a biological reaction. In-vitro studies reported that photobiostimulation accelerates cellular turnover by increasing the expression of osteocalcin [16], stimulating angiogenesis [17], and the availability of mitochondrial ATP [18,19].

In this respect, the present study shows an updated approach to reach a comprehensive diagnosis and efficient orthodontic treatment. In particular, we showed the case of a seven-year-old female affected by transversal maxillary deficiency and anterior openbite. The diagnostic phase included a specific procedure involving mirroring, superimposition, and deviation analysis of 3D models of the maxilla, to detect morphological characteristics of the palatal vault. The therapeutic phase involved the use of photobiomodulation to expedite the correction of crossbite and anterior-openbite.

\section{Materials and Methods}

\subsection{Patient's Clinical Characteristics}

A seven-year-old female attended consultation, having as her chief complaint difficulty in biting and persistent oral breathing pattern. The parents were worried about the negative functional and aesthetic consequences of the presence of an anterior vertical space between upper and lower incisor negative.

Facial examination revealed a eugnatic jaw growing pattern, slight retrusive profile with opened naso-labial angle, and labial competence (Figure 1). Intra-oral clinical examination, recorded with intra-oral photographs (Figure 2), showed early mixed dentition, a $5 \mathrm{~mm}$ anterior openbite, and posterior crossbite at the left side with small loss of coincidence between maxillary and mandibular midlines due to a slight shift of the mandible toward the crossbite side. Both the upper and lower midlines are coincident with the center of maxillary and mandibular arches. Functional examination revealed the presence of infant swallowing pattern with the tongue keeping an anterior position in an attempt to close the anterior space during swallowing or feeding.
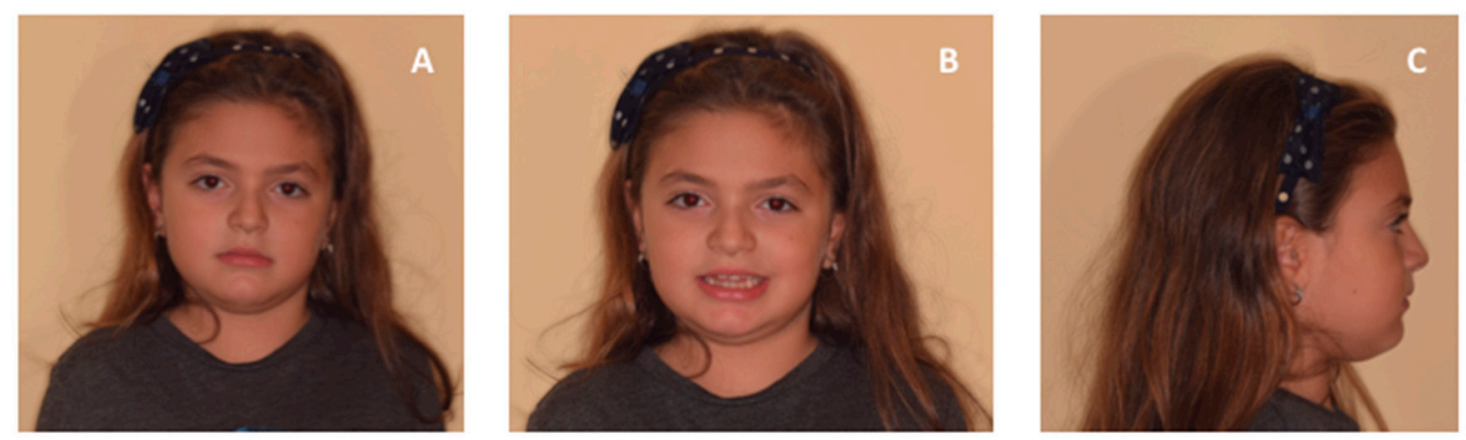

Figure 1. (A-C) Extra-oral facial examination. 

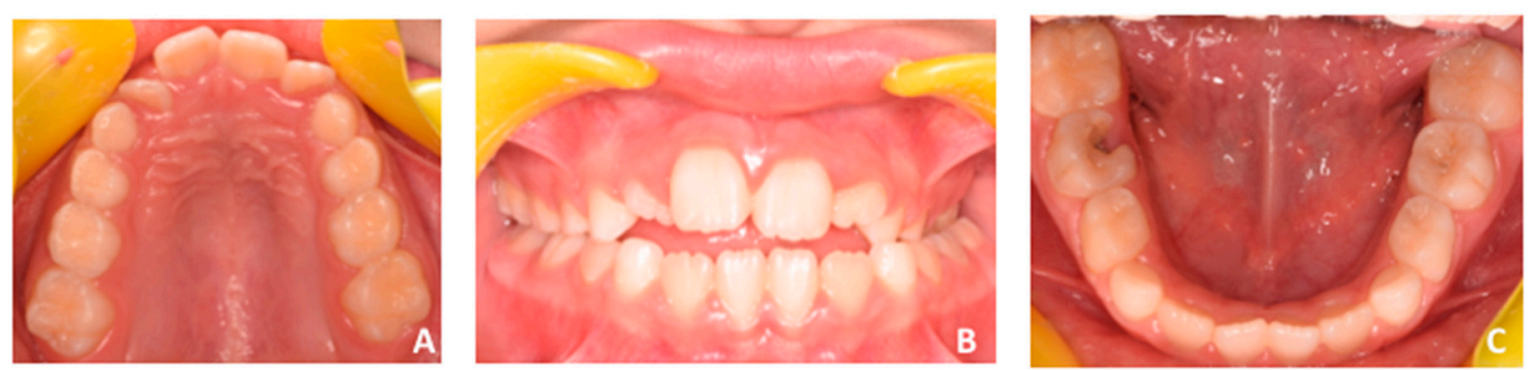

Figure 2. (A-C) Intra-oral examination. See the presence of anterior openbite (A). Additionally, posterior crossbite is present at the left side (B) with loss of coincidence between maxillary and mandibular midlines due to a slight shift of the mandible toward the crossbite side (left side). Both the upper and lower midlines are coincident with the center of maxillary (A) and mandibular (B) arches.

Digital models were registered and integrated within digital articulator in order to better assess the dento-alveolar relationship of both arches in centric occlusion (Figure 3) and centric relation (Figure 4).
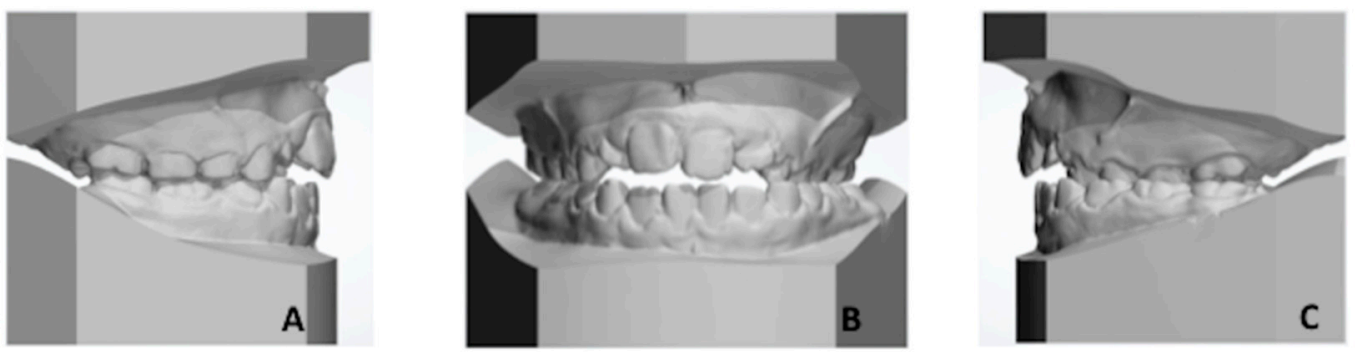

Figure 3. (A-C) Digital models in centric occlusion. (A) Right lateral view. (B) Frontal view shows the posterior crossbite at the left side with loss of coincidence between maxillary and mandibular midlines, due to a slight shift of the mandible toward the left side. (C) Left lateral view.
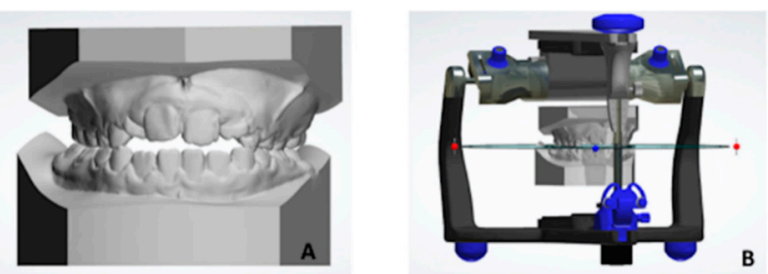

Figure 4. (A) Centric relation, with mandibular and maxillary midlines coincident, showing occlusal interferences leading to mandibular shift upon closure (see intra-oral images). (B) Digital articulator used to simulate centric occlusion of the patient in order to detect occlusal interferences.

Centric occlusion (Figure 3) showed class I relationship on the right side and class II relationship (neutron-occlusion) on the left side. Such discrepancy was attributed to the slight mandibular shift upon closure due to potential occlusal interferences. In fact, when digital models were set in centric relation (Figure 4), i.e., with coincidence of both maxillary and mandibular midlines, posterior occlusal interferences were detected between vestibular cusps of primary molars and first permanent molars. These interferences could be due to a mild maxillary transverse constriction [4].

Panoramic radiograph showed the presence of all permanent dentition with no signs of ectopic eruptive pattern (Figure 5). We did not request lateral cephalogram considering the patient's age and the absence of relevant clinical signs of skeletal antero-posterior discrepancies; thus, useless radiation exposure was avoided for the patient, according to the A.L.A.R.A. principle [20-22]. 


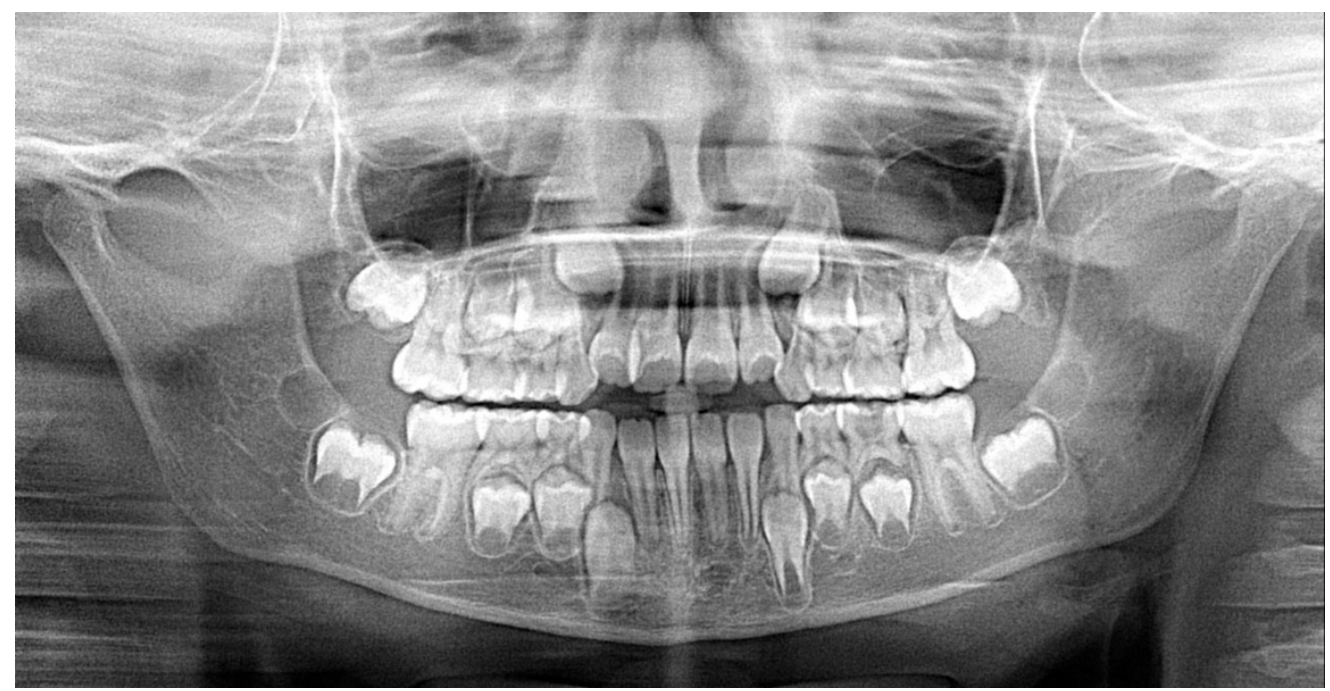

Figure 5. Panoramic radiograph.

To evaluate maxillary morphology and to identify the potential area of maxillary transversal restriction, i.e., palatal vault and/or dento-alveolar process, including the assessment of symmetry or asymmetry, a preliminary digital morphological evaluation was performed by referring to a specific computer-aided workflow.

\subsection{Creation of 3D Virtual Model of Both Maxillary and Mandibular Arches}

Patient received conventional dental impressions using a monophasic polyether impression material (Impregum Penta; '3M ESPE,' Seefeld, Germany) with stainless steel impression trays (Hi-Tray Metal; 'Zhermack SpA,' Rovigo, Italy), which was poured, at most, after 4 hours with type IV stone (Ortostone; Techim Group, Milan, Italy). Then, the maxillary cast was scanned using the D500 3D scanner (3Shape A/S, Copenhagen, Denmark) according to a validated and described system $[5,23]$. After scanning, each dental cast was combined and rendered into a 3D stereo-lithographic model by using a specific software (ScanItOrthodontics ${ }^{\mathrm{TM}} 2015$, version 5.6.1.6, "3Shape A/S," Copenhagen, Denmark).

The digital model of the scanned printed model was exported to Geomagic Qualify software (3D Systems, Rock Hill, Washington, DC, USA) to perform model registration and superimposition and exported to Ortho Analyzer software (3Shape) to perform linear measurements.

\subsection{Digitial Workflow for Anatomical Morphological Evaluation of Maxilla}

A median palatal plane (MPP) was drawn on the maxillary digital cast to identify two landmarks along the median palatal raphe (Figure 6): (1) the point on the median palatal raphe adjacent to the second ruga and (2) the point on the median palatal raphe $1 \mathrm{~cm}$ distal to point one. 


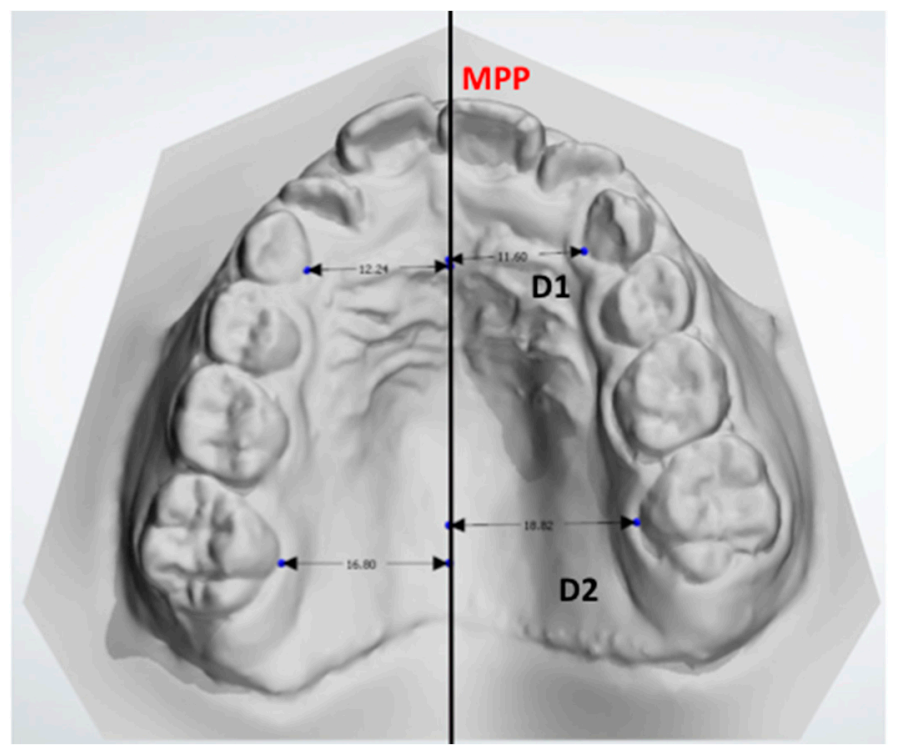

Figure 6. Median palate plane (MPP) drawn on digital maxillary dental arch. See also emi-linear measurements as distance between the MPP and primary canines (D1) and permanent first molars (D2) on both sides.

After identification of MPP, the following measurements were performed (Figure 6):

- D1: the distance between the midpoint at the dento-gingival junction of the primary canine from the crossbite and noncrossbite sides compared with the MPP.

- D2: the distance between the midpoint of the dento-gingival junction of the first molar from the crossbite and noncrossbite sides compared with the MPP.

- D3: the distance between the midpoint at the dento-gingival junction of the two primary canines (Figure 7).

- D4: the distance between the midpoint at the dento-gingival junction of the two first molars (Figure 7).

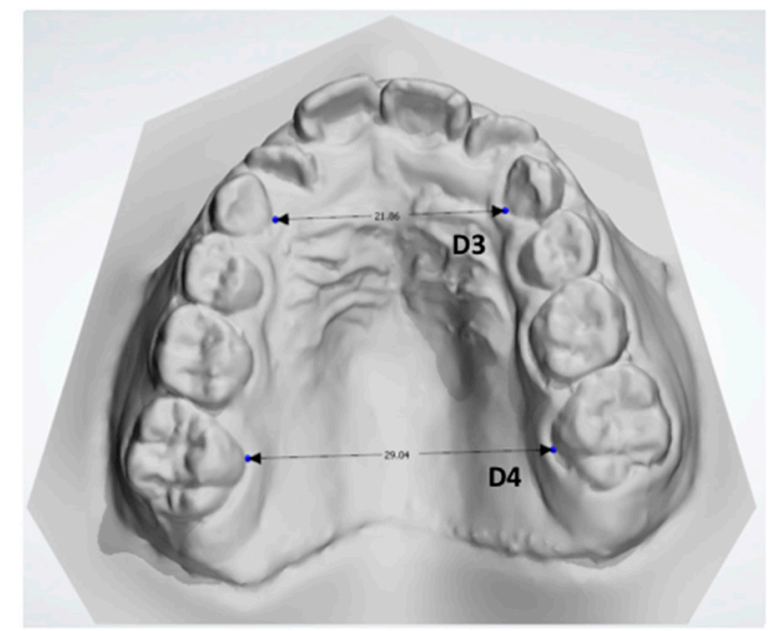

Figure 7. Total linear transversal measurements: distance between the midpoint at the dento-gingival junction of the two primary canines (D3) and the distance between the midpoint at the dento-gingival junction of the two first molars (D4). 
These measurements provide information about the transversal diameter of the maxillary arch (D3-D4) and about the emi-transversal diameters to identify potential linear asymmetry between both sides (D1-D2).

To check for crossbite/noncrossbite symmetry, the digital cast of the patient was superimposed through a semiautomatic surface-to-surface matching technique, using 3D reverse modelling software (Geomagic Control ${ }^{\mathrm{TM}}$ X, version 2017.0.0, '3D Systems,' Rock Hill, SC, USA), which also calculated the deviation between the mirrored and unmirrored 3D palatal models.

- To define the palate surface of the 3D model to be analysed, a gingival plane had to pass through all the most apical points of the dento-gingival junction of all the teeth (from first right molar to first left molar, Figure 8A).
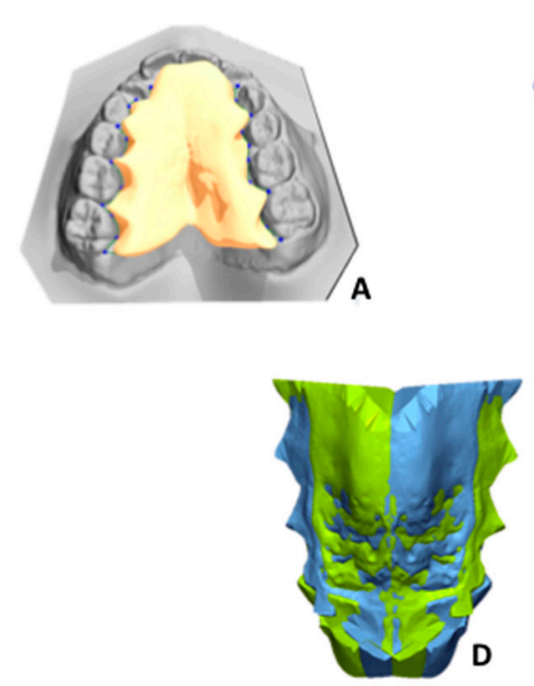
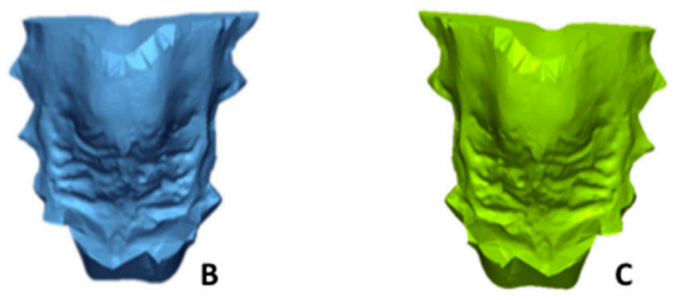

Figure 8. (A-E) The gingival plane (A) was assessed by linking the most apical point of the dento-gingival junction of all teeth at the palatal tooth face. Then the palatal vault model was created (B), mirrored (C), and roughly superimposed using the MPP plane and its perpendicular plane (D). Then, a "best-fit" alignment was done to enhance the superimposition (E) quality.

The workflow for the superimposition of the palate can be divided in four steps, as follows:

- Step 1: Mirroring. Mirroring consists of converting the image orientation from right-left, antero-posterior, and infero-superior to left-right, antero-posterior, and infero-superior (Figure 8B,C).

- Step 2: First registration. Initial manual superimposition of the two models. Pairs of models (the original and the mirrored one of the same patient) were oriented and approximately registered by using the MPP and a line drawn perpendicularly through point two of the MPP. (Figure 8D).

- Step 3. Final registration: Final registration was made using the "best-fit alignment" option in the Geomagic Control X software. The precision of the registration was set to at least $0.3 \mathrm{~mm}$ (tolerance type: “3D Deviation") with a maximum of 100,000 polygons for surface representation. The corresponding polygons from selected reference areas were automatically superimposed (Figure 8E).

- Step 4. Superimposition and 3D analysis: The distances between corresponding areas of the original maxillary cast and the corresponding mirrored one were compared to obtain color-coded maps (Figure 9). The yellow-to-red fields indicated that the definitive casts were larger than the master model, and the turquoise-to-dark blue fields indicated that the definitive casts were smaller than the master model. The 3D deviation analysis has a tolerance range (green) of $\pm 0.50 \mathrm{~mm}$ with a maximum of $2 \mathrm{~mm}$. All the values in this range indicated the matching percentage between the two specular 3D models. 


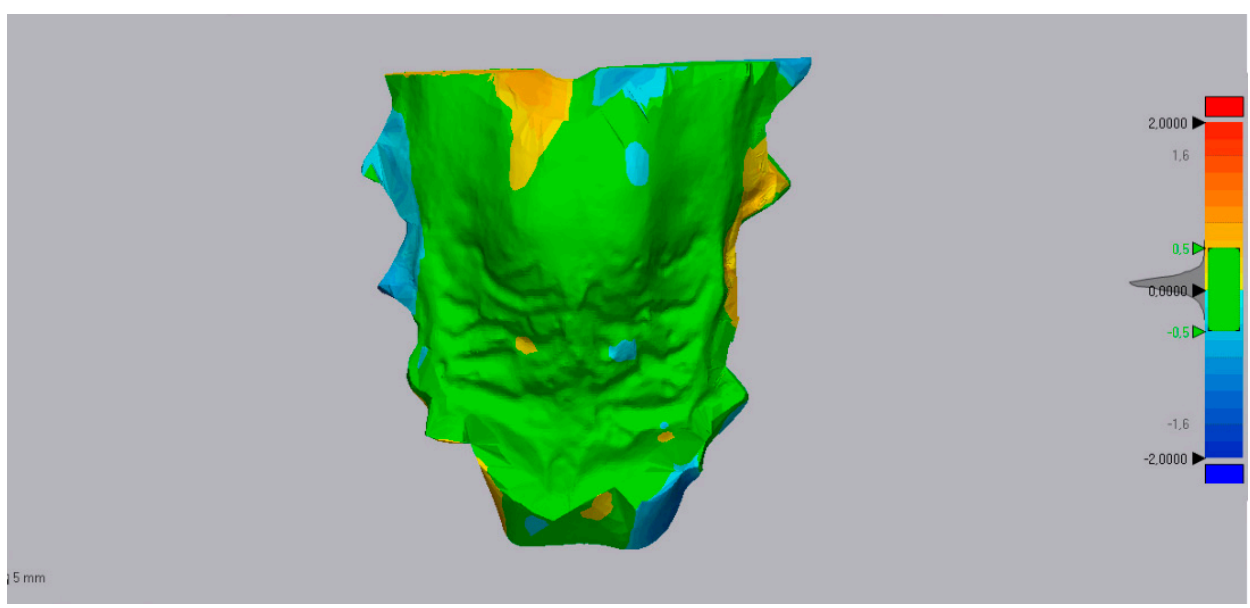

Figure 9. 3D deviation analysis between the two specular models. RGB colored scale bar (millimeters) is reported on the right side: the top (red colored) and the bottom (blue colored) of the scale indicate total mismatching. Green indicates matching percentage. As shown, both palatal shells (original and mirrored) were fairly matched. Some amount of mismatching (light blue and orange contours) were located along the cervical contour of posterior teeth, due to dento-alveolar positional differences.

\subsection{Diagnosis and Treatment Plan}

Transversal measurements of the maxilla showed a distance of $21.86 \mathrm{~mm}$ between primary canines and $29.04 \mathrm{~mm}$ between primary second molars. According to the study of McNamara and coauthors [4,24], a clinical value of inter-molar distances $\leq 31 \mathrm{~mm}$ should be considered as a cut-off parameter for diagnosis of narrowed maxillary requiring treatment by maxillary expansion. However, considering that the patient was only 6 years old and that she presented full primary dentition, this cut-off could be higher considering the potential growth of the patient [24]. Thus, the maxillary contraction could be considered as moderate in severity.

Emi-lateral distances to the MPP at the primary canines level were $12.24 \mathrm{~mm}$ at the right side and $11.60 \mathrm{~mm}$ at the left side. The same distances at the first molars level were $16.80 \mathrm{~mm}$ at the right side and $16.82 \mathrm{~mm}$ at the left side. Thus, no relevant differences were detected between right and left side concerning transversal linear measurements. Additionally, the deviation analysis of mirrored superimposed models of the maxilla revealed a good coincidence between the two models, as shown by the the colored map (green color). In particular, Figure 9 shows no areas of mismatching (intense blue and intense red contours) between the original and specular model either in the palatal vault or along the profile of the dento-alveolar processes.

Thus, according to the diagnostic information obtained, the patient presented a slight bilateral symmetric contraction of the palatal vault with symmetric contraction of the alveolar process of the upper arch. As a consequence, our treatment goal was to correct the maxillary transverse deficiency by symmetrically expanding both the palatal vault and dento-alveolar processes, eliminating those occlusal interferences causing an altered posture of the mandible (mandibular shift). Along with this outcome, it was imperative to correct the anterior openbite in order to restore a correct functional posture of the tongue during swallowing and biting.

We decided to construct a maxillary removable mono-block with a built-in Hyrax screw and with an anterior grid that was extended 3-4 mm below the lower incisor's marginal edge. This grid forced the tongue in a more physiologic position (backward and upward), with the tip of the tongue contacting the retro-incisal papilla (Figure $10 \mathrm{~A}, \mathrm{~B}$ ). With the tongue in a more backward position, upper and lower incisor were left free to erupt facilitating the closure of the bite [25], without the application of orthodontic forces on these teeth. The screw was slowly activated, i.e., two times a week up to the clinical assessment of crossbite resolution and correction of mandible posture in centric relation, with both maxillary and mandibular midlines coincident. 

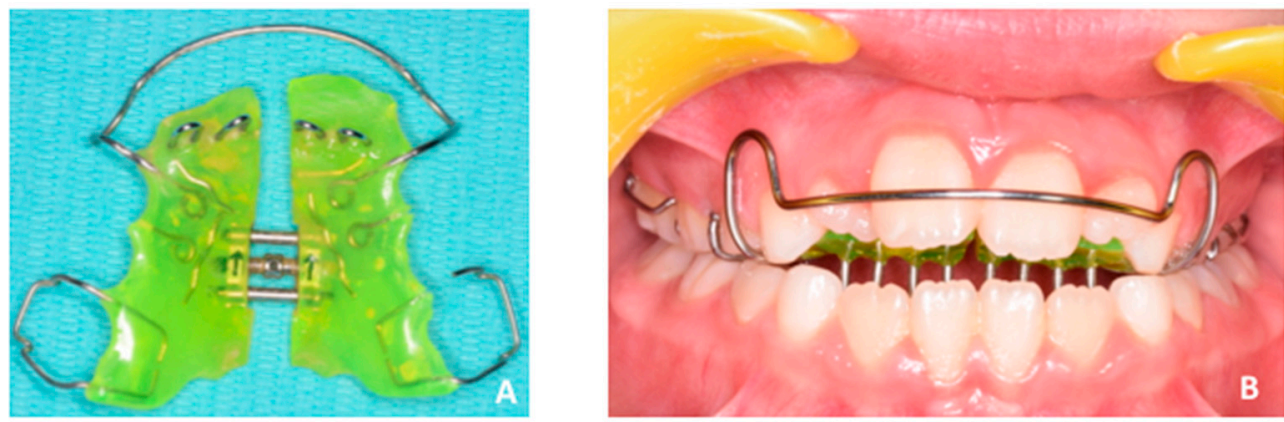

Figure 10. (A,B) Removable appliance with built-in Hyrax screw and asymmetric cut of the resin in order to provide bilateral expansion of the maxilla (A). Frontal intra-oral photograph showing the anterior grid behind upper and lower incisors (B).

\subsection{PBM Administration}

PBM was administered using a Diode laser emitting infra-red radiation at $980 \mathrm{~nm}$ (Wiser, Doctor Smile—Lambda Spa, Brendola, VI). The flat top optical fiber (AB 2799, Doctor Smile—Lambda Spa, Brendola, VI) dispensed a beam spot size of $1 \mathrm{~cm}^{2}$, and irradiation was administered by positioning the optical fiber tip (1.5 $\mathrm{cm}$ as minimum on defocalization, as prescribed by the producer) (1) along the MPP and (2) along the anterior upper and lower dental segment (canine to canine). All irradiations were done with an output power of $1 \mathrm{~W}$ at a continuous wave. The palate was irradiated in three points, i.e., the area behind the first palatal ruga, the area behind the third palatal ruga, and the mesial area of soft palate. Each area was irradiated one time for $50 \mathrm{~s}$, with an interval of $2 \mathrm{~min}$ between each administration, i.e., for a total energy density of $150 \mathrm{~J} / \mathrm{cm}^{2}$. Four dental segments (deciduous right first molar-canine, right lateral-central incisors, left-central lateral incisors, deciduous left canine-first premolar) were consecutively irradiated for $6 \mathrm{~s}$, for a total of $24 \mathrm{~s}$, in both maxillary and mandibular arches. The procedure was repeated three times with an interval of $2 \mathrm{~min}$ between each administration, for a total energy density of $72 \mathrm{~J} / \mathrm{cm}^{2}$ for each jaw.

\section{Results}

Figures 11 and 12 show patient's occlusal characteristics 4 months after treatment. Maxillary transverse deficiency was corrected with complete resolution of the unilateral crossbite on the left side. Patient shows bilateral class I occlusion with coincidence of both midlines upon closure (Figure 12). The anterior bite was completely closed, restoring a physiological functional pattern during biting and swallowing. Additionally, the aesthetics of the patient significantly improved, especially while smiling (Figure 11). The total active expansion treatment time was two months, while the same appliance was worn longer in order to obtain correction of the openbite.
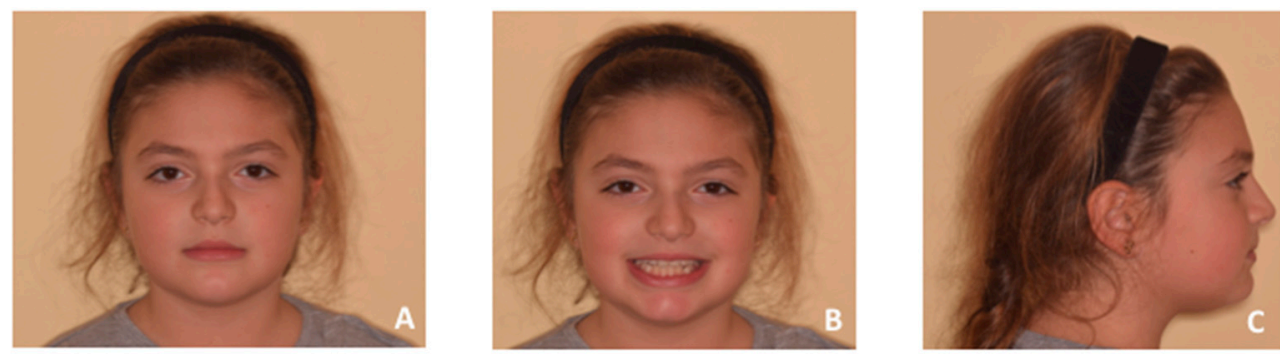

Figure 11. (A-C) Post treatment extra-oral examination. 

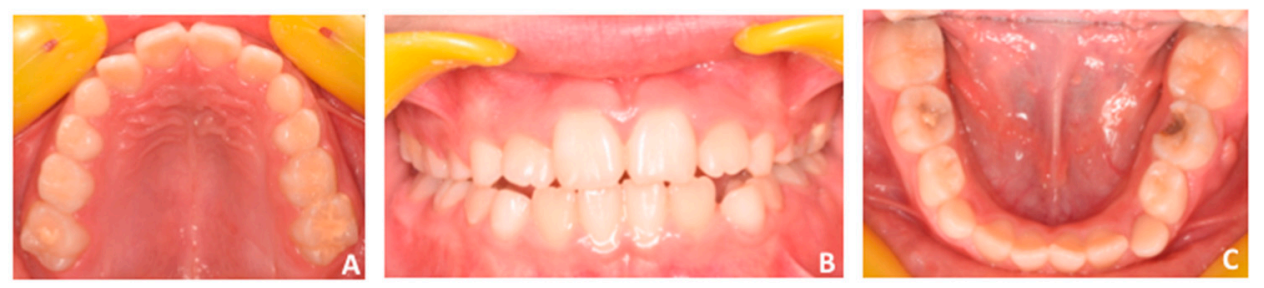

Figure 12. (A-C) Intra-oral examination. In particular, see the absence of crossbite at the right side (A) and the coincidence between maxillary and mandibular midlines (B).

Post-treatment radiographic examination such as dental panorex was not required considering the young age of the patient and the short overall treatment time (4 months), thus avoiding useless radiation according to the A.L.A.R.A. principle [20-23].

\section{Discussion}

The present paper shows how new technologies can be integrated with acquired knowledge and skills of orthodontic clinicians in order to enhance the diagnosis and treatment plan of specific malocclusion. We discussed the treatment of a seven-year-old female presenting transverse maxillary deficiency and anterior openbite, and we proposed two distinct methods involving (1) the use of a digital diagnostic workflow for the thorough evaluation of maxillary morphology and (2) the use of photobiomodulation to expedite dental movement. Both methodologies have been described in previous studies $[5,10,12,13]$.

We evaluated the palatal size and morphology of a seven-year-old female affected by unilateral functional crossbite patients by using digital linear assessment of maxillary transversal diameters and by surface-based superimposition of mirrored maxillary models. According to this reverse engineering, each virtual palate can be mirrored at an arbitrary point. This procedure allows for the detection of morphologic differences between the two emi-palatal halves. Moreover, the 3D differences of the registered models are usually translated into color codes that represent the distance between corresponding points $[25,26]$.

By this method, we can verify that the maxilla was narrowed with a symmetric growth pattern involving both the palatal vault and the dento-alveolar processes (Figure 9). This is in disagreement with previous findings showing asymmetric transverse growth of the dento-alveolar process in presence of functional unilateral-posterior crossbite in children [5,27-31]. A possible explanation could be that adaptative asymmetric changes of the shape of the dento-alveolar process have still not occurred in the patient presented here. In a previous study [6], the same reverse engineering methodology was used to detect areas of asymmetry in the maxilla of a patient affected by MTD. Authors concluded that reverse engineering methodology could help clinicians in developing a specific appliance for asymmetric expansion of the maxilla. Here, according to the information provided by deviation analysis, we needed to expand the maxilla symmetrically, confirming that the presented diagnostic digital workflow is helpful in providing a comprehensive assessment of the morphology of the palate.

Treatment outcomes were reached in less then 4 months. In particular, the anterior openbite was corrected by the combination of the lingual grid and PBM [27-29]. While the anterior grid forced the tongue in backward and upward position, anterior teeth were free to erupt since they were not obstructed by tongue interposition [30,32]. This spontaneous eruption was expedited by the administration of PBM, which is able to provoke an accelerated metabolic activity by increasing cellular turnover [13], increasing vascular activity $[14,33,34]$, and the availability of ATP $[15,35,36]$. In this respect, PBM is a free-side effects procedure that has also been validated for several therapeutic applications in children $[13,37,38]$.

PBM can create a favorable environment for tooth movement, as two types of host responses occur in the irradiated tissues. First, photobiomodulation increases the production of mitochondrial ATP by upregulating the cytochrome c oxidase $[12,14,39-45]$ and promotes the cellular viability and the 
expression of osteocalcin in the tension areas of PDL [12-15,39-43]. This increased metabolic activity accelerates cellular turnover (osteoclast, osteoblast, and fibroblasts) [44-53] and the production of cytokines involved in the bone remodeling, mainly the IL-1b [46,54-57]. Second, photobiomodulation activates the receptor of nuclear factor kappa B (RANK) and macrophage-colony stimulating factor along with its receptor (c-fms) that, respectively, seem to play a role in the expedition of dental movement [44].

\section{Limitations}

The present study was designed as a case report to fully document a digital orthodontic diagnostic workflow and specific clinical treatment procedure. However, further studies with appropriate sample size, including both patients and orthodontists, are required in order to assess if the presented digital workflow can increase the effectiveness and the comprehensiveness of the diagnostic process.

Author Contributions: Conceptualization, V.Q. and A.L.G. methodology, A.L.G..; software, V.R.; validation, V.C. and A.L.G.; formal analysis, S.T.; investigation, L.M.; writing—original draft preparation, A.L.G. writing-review and editing, V.Q. and A.L.G.; visualization, V.R. and A.L.G.; All authors have read and agreed to the published version of the manuscript.

Funding: This research received no external funding.

Conflicts of Interest: The authors declare no conflict of interest.

\section{References}

1. Silva Filho, O.G.; Santamaria, M.; Jrand Capelozza Filho, L. Epidemiology of posterior crossbite in the primary dentition. J. Clin. Pediatr. Dent. 2007, 32, 73-78. [CrossRef] [PubMed]

2. Quinzi, V.; Rossi, O.; Paglia, L.; Marzo, G.; Caprioglio, A. Paediatric Orthodontics Part 2: Periodontal effects of maxillary expansion. Eur. J. Paediatr. Dent. 2019, 20, 164-166. [PubMed]

3. Lo Giudice, A.; Fastuca, R.; Portelli, M.; Militi, A.; Bellocchio, M.; Spinuzza, P.; Briguglio, F.; Caprioglio, A.; Nucera, R. Effects of rapid vs slow maxillary expansion on nasal cavity dimensions in growing subjects: A methodological and reproducibility study. Eur. J. Paediatr. Dent. 2017, 18, 299-304. [PubMed]

4. McNamara, J.A. Maxillary transverse deficiency. Am. J. Orthod. Dentofac. Orthop. 2000, 117, 567-570. [CrossRef]

5. Leonardi, R.; Lo Giudice, A.; Rugeri, M.; Muraglie, S.; Cordasco, G.; Barbato, E. Three-dimensional evaluation on digital casts of maxillary palatal size and morphology in patients with functional posterior crossbite. Eur. J. Orthod. 2018, 40, 556-562. [CrossRef]

6. Isola, G.; Anastasi, G.; Matarese, G.; Williams, R.C.; Cutroneo, G.; Bracco, P.; Piancino, M.G. Functional and molecular outcomes of the human masticatory muscles. Oral Dis. 2018, 24, 1428-1441. [CrossRef]

7. Primožic, J.; Baccetti, T.; Franchi, L.; Richmond, S.; Farčnik, F.; Ovsenik, M. Three-dimensional assessment of palatal change in a controlled study of unilateral posterior crossbite correction in the primary dentition. Eur. J. Orthod. 2013, 35, 199-204. [CrossRef]

8. Ganzer, N.; Feldmann, I.; Liv, P.; Bondemark, L. A novel method for superimposition and measurements on maxillary digital 3D models-studies on validity and reliability. Eur. J. Orthd. 2017, 40, 45-51. [CrossRef]

9. Kapila, S.D.; Nervina, J.M. CBCT in orthodontics: Assessment of treatment outcomes and indications for its use. Dentomaxillofac. Radiol. 2015, 44, 20140282. [CrossRef]

10. Leonardi, R.; Muraglie, S.; Lo Giudice, A.; Aboulazm, K.S.; Nucera, R. Evaluation of mandibular symmetry and morphology in adult patients with unilateral posterior crossbite. A CBCT study using a surface-to-surface matching technique. Eur. J. Orthod. 2020. [Epub ahead of print]. [CrossRef]

11. Lo Giudice, A.; Brewer, I.; Leonardi, R.; Roberts, N.; Bagnato, G. Pain threshold and temporomandibular function in systemic sclerosis: Comparison with psoriatic arthritis. Clin. Rheumatol. 2018, 37, 1861-1867. [CrossRef] [PubMed]

12. Lo Giudice, A.; Nucera, R.; Leonardi, R.; Paiusco, A.; Baldoni, M.; Caccianiga, G. A comparative assessment of the efficiency of orthodontic treatment with and without photobiomodulation during mandibular de-crowding in young subjects: A single-center, single blind randomized controlled trial. Photobiomodul Photomed. Laser Surg. 2020. [Epub ahead of print]. [CrossRef] [PubMed] 
13. Lo Giudice, A.; Nucera, R.; Perillo, L.; Paiusco, A.; Caccianiga, G. Is Low-Level Laser Therapy an Effective Method to Alleviate Pain Induced by Active Orthodontic Alignment Archwire? A Randomized Clinical Trial. J. Evid. Based Dent. Pract. 2019, 19, 71-78. [CrossRef]

14. Caccianiga, G.; Crestale, C.; Cozzani, M.; Piras, A.; Mutinelli, S.; Lo Giudice, A.; Cordasco, G. Low-level laser therapy and invisible removal aligners. J. Biol. Regul. Homeost. Agents 2016, 30 (Suppl. 1), 107-113.

15. Caccianiga, G.; Paiusco, A.; Perillo, L.; Nucera, R.; Pinsino, A.; Maddalone, M.; Cordasco, G.; Lo Giudice, A. Does low-level laser therapy enhance the efficiency of orthodontic dental alignment? Results from a randomized pilot study. Photomed. Laser Surg. 2017, 35, 421-426. [CrossRef] [PubMed]

16. Huang, T.H.; Liu, S.L.; Chen, C.L.; Shie, M.Y.; Kao, C.T. Low-level laser effects on simulated orthodontic tension side periodontal ligament cells. Photomed. Laser Surg. 2013, 31, 72-77. [CrossRef]

17. Eells, J.T.; Henry, M.M.; Summerfelt, P.; Wong-Riley, M.T.; Buchmann, E.V.; Kane, M.; Whelan, N.T.; Whelan, H.T. Therapeutic photobiomodulation for methanol-induced retinal toxicity. Proc. Natl. Acad. Sci. USA 2003, 100, 3439-3444. [CrossRef]

18. Oron, U.; Ilic, S.; De Taboada, L.; Streeter, J. Ga-As (808 nm) laser irradiation enhances ATP production in human neuronal cells in culture. Photomed. Laser Surg. 2007, 25, 180-182. [CrossRef]

19. Paduano, F.; Marrelli, M.; Alom, N.; Amer, M.; White, L.J.; Shakesheff, K.M.; Tatullo, M. Decellularized bone extracellular matrix and human dental pulp stem cells as a construct for bone regeneration. J. Biomater. Sci. Polym. Ed. 2017, 28, 730-748. [CrossRef]

20. Isola, G.; Matarese, M.; Ramaglia, L.; Cicciù, M.; Matarese, G. Evaluation of the efficacy of celecoxib and ibuprofen on postoperative pain, swelling, and mouth opening after surgical removal of impacted third molars: A randomized, controlled clinical trial. Int. J. Oral Maxillofacial Surg. 2019, 48, 1348-1354. [CrossRef]

21. Lo Giudice, A.; Caccianiga, G.; Crimi, S.; Cavallini, C.; Leonardi, R. Frequency and type of ponticulus posticus in a longitudinal sample of nonorthodontically treated patients: Relationship with gender, age, skeletal maturity, and skeletal malocclusion. Oral Surg. Oral Med. Oral Pathol. Oral Radiol. 2018, 126, 291-297. [CrossRef] [PubMed]

22. Nucera, R.; Lo Giudice, A.; Bellocchio, M.; Spinuzza, P.; Caprioglio, A.; Cordasco, G. Diagnostic concordance between skeletal cephalometrics, radiograph-based soft-tissue cephalometrics, and photograph-based soft-tissue cephalometrics. Eur. J. Orthod. 2017, 39, 352-357. [CrossRef] [PubMed]

23. Isola, G.; Perillo, L.; Migliorati, M.; Matarese, M.; Dalessandri, D.; Grassia, V.; Alibrandi, A.; Matarese, G. The impact of temporomandibular joint arthritis on functional disability and global health in patients with juvenile idiopathic arthritis. Eur. J. Orthod. 2019, 29, 117-124. [CrossRef] [PubMed]

24. Massaro, C.; Losada, C.; Cevidanes, L.; Yatabe, M.; Garib, D.; Lauris, J.R.P.; Ioshida, M.; Rey, D.; Alvarez, M.A.; Benavides, E.; et al. Comparison of linear and angular changes assessed in digital dental models and cone-beam computed tomography. Orthod. Craniofac. Res. 2019, 23, 118-128. [CrossRef] [PubMed]

25. Maurice, T.J.; Kula, K. Dental arch asymmetry in the mixed dentition. Angle Orthod. 1998, 68, 37-44. [PubMed]

26. Leonardi, R. Cone-beam computed tomography and three-dimensional orthodontics. Where we are and future perspectives. J. Orthod. 2019, 46 (Suppl. 1), 45-48. [CrossRef]

27. Maspero, C.; Abate, A.; Bellincioni, F.; Cavagnetto, D.; Lanteri, V.; Costa, A.; Farronato, M. Comparison of a tridimensional cephalometric analysis performed on 3T-MRI compared with CBCT: A pilot study in adults. Prog. Orthod. 2019, 20, 40. [CrossRef]

28. Isola, G.; Matarese, G.; Ramaglia, L.; Pedullà, E.; Rapisarda, E.; Iorio-Siciliano, V. Association between periodontitis and glycosylated haemoglobin before diabetes onset: A cross-sectional study. Clin. Oral Investig. 2019. [CrossRef]

29. Piancino, M.G.; Isola, G.; Cannavale, R.; Cutroneo, G.; Vermiglio, G.; Bracco, P.; Anastasi, G.P. From periodontal mechanoreceptors to chewing motor control: A systematic review. Arch. Oral Biol. 2017, 78, 109-121. [CrossRef]

30. Piancino, M.G.; Di Benedetto, L.; Matacena, G.; Deregibus, A.; Marzo, G.; Quinzi, V. Paediatric Orthodontics Part 3: Masticatory function during development. Eur. J. Paediatr. Dent. 2019, 20, 247-249.

31. Lo Giudice, A.; Barbato, E.; Cosentino, L.; Ferraro, C.M.; Leonardi, R. Alveolar bone changes after rapid maxillary expansion with tooth-born appliances: A systematic review. Eur. J. Orthod. 2018, 40, 296-303. [CrossRef] [PubMed] 
32. Rosa, M.; Quinzi, V.; Marzo, G. Paediatric Orthodontics Part 1: Anterior open bite in the mixed dentition. Eur. J. Paediatr. Dent. 2019, 20, 80-82. [PubMed]

33. Isola, G.; Polizzi, A.; Muraglie, S.; Leonardi, R.; Lo Giudice, A. Assessment of Vitamin C and Antioxidant Profiles in Saliva and Serum in Patients with Periodontitis and Ischemic Heart Disease. Nutrients 2019, 11, 2956. [CrossRef] [PubMed]

34. Isola, G.; Polizzi, A.; Santonocito, S.; Alibrandi, A.; Ferlito, S. Expression of Salivary and Serum Malondialdehyde and Lipid Profile of Patients with Periodontitis and Coronary Heart Disease. Int. J. Mol. Sci. 2019, 20, 6061. [CrossRef] [PubMed]

35. Isola, G.; Alibrandi, A.; Currò, M.; Matarese, M.; Ricca, S.; Matarese, G.; Ientile, R.; Kocher, T. Evaluation of salivary and serum ADMA levels in patients with periodontal and cardiovascular disease as subclinical marker of cardiovascular risk. J. Periodontol. 2020. [CrossRef] [PubMed]

36. González Espinosa, D.; Santos, M.; Mendes, S.M.D.A.; Normando, D. Mandibular propulsion appliance for adults with Class II malocclusion: A systematic review and meta-analysis. Eur. J. Orthod. 2019, cjz089. [CrossRef]

37. Muniz, R.S.C.; Carvalho, C.N.; Aranha, A.C.C.; Dias, F.M.C.S.; Ferreira, M.C. Efficacy of low-level laser therapy associated with fluoride therapy for the desensitisation of molar-incisor hypomineralisation: Randomised clinical trial. Int. J. Paediatr. Dent. 2019. [Epub ahead of print]. [CrossRef]

38. Kobayashi, F.Y.; Castelo, P.M.; Gonçalves, M.L.L.; Motta, L.J.; Mota, A.C.D.C.; Altavista, O.M.; Pinto, M.M.; Salgueiro, M.C.; Ferreira, K.P.S.; Bussadori, S.K. Evaluation of the effectiveness of infrared light-emitting diode photobiomodulation in children with sleep bruxism: Study protocol for randomized clinical trial. Medicine 2019, 98, e17193. [CrossRef]

39. Caccianiga, G.; Lo Giudice, A.; Paiusco, A.; Portelli, M.; Militi, A.; Baldoni, M.; Nucera, R. Maxillary Orthodontic Expansion Assisted by Unilateral Alveolar Corticotomy and Low-Level Laser Therapy: A Novel Approach for Correction of a Posterior Unilateral Cross-Bite in Adults. J. Lasers Med. Sci. 2019, 10, $225-229$. [CrossRef]

40. Lo Giudice, G.; Lo Giudice, A.; Isola, G.; Fabiano, F.; Artemisia, A.; Fabiano, V.; Nucera, R.; Matarese, G. Evaluation of bond strength and detachment interface distribution of different bracket base designs. Acta Med. Mediterr 2015, 31, 585.

41. Nucera, R.; Militi, A.; Lo Giudice, A.; Longo, V.; Fastuca, R.; Caprioglio, A.; Cordasco, G.; Papadopoulos, M.A. Skeletal and Dental Effectiveness of Treatment of Class II Malocclusion With Headgear: A Systematic Review and Meta-analysis. J. Evid. Based Dent. Pract. 2018, 18, 41-58. [CrossRef] [PubMed]

42. Traini, T.; Danza, M.; Zollino, I.; Altavilla, R.; Lucchese, A.; Sollazzo, V.; Trapella, G.; Brunelli, G.; Carinci, F. Histomorphometric evaluation of an immediately loaded implant retrieved from human mandible after 2 years. Int. J. Immunopathol. Pharmacol. 2011, 24, 31-36. [CrossRef] [PubMed]

43. Pastorino, R.; Gherlone, E.F.; Perillo, L.; Lupi, S.M.; Lucchese, A. Histomorphometric evaluation of two different bone substitutes in sinus augmentation procedures: A randomized controlled trial in humans. Int. J. Oral Maxillofac. Implants 2017, 32, 188-194.

44. Isola, G.; Alibrandi, A.; Pedullà, E.; Grassia, V.; Ferlito, S.; Perillo, L.; Rapisarda, E. Analysis of the effectiveness of Lornoxicam and Flurbiprofen on management of pain and sequelae following third molar surgery: A randomized, controlled, clinical trial. J. Clin. Med. 2019, 8, 325. [CrossRef] [PubMed]

45. Isola, G.; Matarese, M.; Ramaglia, L.; Iorio-Siciliano, V.; Cordasco, G.; Matarese, G. Efficacy of a drug composed of herbal extracts on postoperative discomfort after surgical removal of impacted mandibular third molar: A randomized, triple-blind, controlled clinical trial. Clin. Oral Investig. 2019, 23, 2443-2453. [CrossRef] [PubMed]

46. Isola, G.; Polizzi, A.; Alibrandi, A.; Indelicato, F.; Ferlito, S. Analysis of Endothelin-1 concentrations in individuals with periodontitis. Sci. Rep. 2020. [CrossRef]

47. Isola, G.; Alibrandi, A.; Rapisarda, E.; Matarese, G.; Williams, R.C.; Leonardi, R. Association of vitamin d in patients with periodontal and cardiovascular disease: A cross-sectional study. J. Periodontal Res. 2020, in press.

48. Spagnuolo, G.; Ametrano, G.; D’Antò, V.; Rengo, C.; Simeone, M.; Riccitiello, F.; Amato, M. Effect of autoclaving on the surfaces of TiN -coated and conventional nickel-titanium rotary instruments. Int. Endod. J. 2012, 45, 1148-1155. [CrossRef] 
49. Spagnuolo, G.; Ametrano, G.; D’Antò, V.; Formisano, A.; Simeone, M.; Riccitiello, F.; Amato, M.; Rengo, S. Microcomputed tomography analysis of mesiobuccal orifices and major apical foramen in first maxillary molars. Open Dent. J. 2012, 6, 118-125. [CrossRef]

50. Ametrano, G.; D'Antò, V.; Di Caprio, M.P.; Simeone, M.; Rengo, S.; Spagnuolo, G. Effects of sodium hypochlorite and ethylenediaminetetraacetic acid on rotary nickel-titanium instruments evaluated using atomic force microscopy. Int. Endod. J. 2011, 44, 203-209. [CrossRef]

51. Cavuoti, S.; Matarese, G.; Isola, G.; Abdolreza, J.; Femiano, F.; Perillo, L. Combined orthodontic-surgical management of a transmigrated mandibular canine: A case report. Angle Orthod. 2016, 86, 681-691. [CrossRef] [PubMed]

52. Currò, M.; Matarese, G.; Isola, G.; Caccamo, D.; Ventura, V.P.; Cornelius, C.; Lentini, M.; Cordasco, G.; Ientile, R. Differential expression of transglutaminase genes in patients with chronic periodontitis. Oral Dis. 2014, 20, 616-623. [CrossRef]

53. Matarese, G.; Isola, G.; Alibrandi, A.; Lo Gullo, A.; Bagnato, G.; Cordasco, G.; Perillo, L. Occlusal and MRI characterizations in systemic sclerosis patients: A prospective study from Southern Italian cohort. Jt. Bone Spine 2016, 83, 57-62. [CrossRef] [PubMed]

54. Matarese, G.; Currò, M.; Isola, G.; Caccamo, D.; Vecchio, M.; Giunta, M.L.; Ramaglia, L.; Cordasco, G.; Williams, R.C.; Ientile, R. Transglutaminase 2 up-regulation is associated with RANKL/OPG pathway in cultured HPDL cells and THP-1-differentiated macrophages. Amino Acids 2015, 47, 2447-2455. [CrossRef] [PubMed]

55. Isola, G.; Lo Giudice, A.; Polizzi, A.; Alibrandi, A.; Patini, R.; Ferlito, S. Periodontitis and Tooth Loss Have Negative Systemic Impact on Circulating Progenitor Cell Levels: A Clinical Study. Genes 2019, 7, 1022. [CrossRef]

56. Varella, A.M.; Revankar, A.V.; Patil, A.K. Low-level laser therapy increases interleukin-1 $\beta$ in gingival crevicular fluid and enhances the rate of orthodontic tooth movement. Am. J. Orthod. Dentofac. Orthop. 2018, 154, 535-544. [CrossRef]

57. Farronato, M.; Maspero, C.; Lanteri, V.; Fama, A.; Ferrati, F.; Pettenuzzo, A.; Farronato, D. Current state of the art in the use of augmented reality in dentistry: A systematic review of the literature. BMC Oral Health 2019, 19, 135. [CrossRef] 